\title{
Síndrome Metabólica em Mulheres Com Síndrome dos Ovários Policísticos: Prevalência em Cidade da Região Nordeste do Brasil
}

\section{Sr. Editor,}

OM bastante INTERESSE, vimos o artigo de Silva e cols. intitulado "Síndrome dos ovários policísticos, síndrome metabólica, risco cardiovascular e o papel dos agentes sensibilizadores da insulina", recentemente publicado nos ABE\&M (1). Com base em resultados referentes à população americana de mulheres com síndrome dos ovários policísticos (SOP), os autores destacam que a prevalência de síndrome metabólica (SM) nesse grupo varia de 33 a $43 \%$, sendo cerca de duas vezes maior do que a taxa observada em mulheres da população geral, não fazendo referência, entretanto, à prevalência de SM em mulheres brasileiras com SOP (1).

Estudos realizados em populações não-americanas mostram resultados conflitantes, onde a prevalência de SM na SOP variou de 1,6 a 16\%, respectivamente na República Tcheca (2) e na Itália (3). Tais inconsistências podem ser atribuídas tanto a fatores genéticos quanto a diferenças nos hábitos alimentares e na prevalência de obesidade entre as populações, assim como a vieses metodológicos decorrentes dos critérios empregados para diagnosticar SOP e SM, nos diversos estudos publicados.

Em relação à população brasileira, são poucos os relatos publicados sobre a prevalência de SM em mulheres portadoras da SOP. Neste sentido, realizamos estudo com o objetivo de determinar a prevalência de SM numa amostra de 102 mulheres com diagnóstico de SOP de acordo com o Consenso de Rotterdam (4), residentes em Natal, RN. A prevalência de SM conforme os critérios do NCEP ATPIII (5) foi de 28,4\%, sendo crescente de acordo com a faixa etária $(10,0 \%$ de $20-24$ anos, $34,2 \%$ de 25-29 anos e 46,2\% de 30-34anos; p < 0,05). Apesar de não existirem relatos detalhados acerca da prevalência de SM na população geral brasileira, segundo sexo e idade, ao compararmos nossos dados com resultados referentes à prevalência de $\mathrm{SM}$ em área rural do semi-árido baiano (6), observamos que a taxa detectada nas pacientes com SOP é bastante superior àquela verificada para pessoas na faixa etária entre 25 e 34 anos $(7,0 \%)$. No que diz respeito à $\mathrm{SOP}$, existem apenas resultados referentes a uma comunicação em recente evento científico de âmbito internacional, apontando prevalência de 38,4\% em mulheres residentes no Estado de São Paulo (7). Considerando-se as dimensões continentais de nosso país, é plausível admitir que fatores genéticos, ambientais e socioeconômicos podem ser responsáveis por diferenças regionais na prevalência não apenas da SM, mas também de outras morbidades cardiovasculares, como hipertensão arterial e diabetes mellitus.

Em nossa casuística, um percentual de $85,3 \%$ das mulheres com SOP apresentou, no mínimo, uma anormalidade componente da SM, o que vem demonstrar a relevância de seu rastreamento em todas as portadoras de SOP. De acordo com a quantidade de critérios presentes, foi observada a seguinte distribuição: nenhum critério $(\mathrm{n}=15 ; 14,7 \%), \mathrm{l}(\mathrm{n}=$ $27 ; 26,5 \%), 2(n=31 ; 30,4 \%), 3(n=20 ; 19,6 \%)$ e $4(n=9 ; 8,8 \%)$. Dentre

\section{carta ao editor}

\author{
ELVIRA M.M. SOARES \\ GEORGE D. AZEVEDO \\ TECIA M.O. MARANHÃO
}

Programa de Pós-graduação em Ciências da Saúde (EMMS, GDA \& TMOM), Departamentos de Morfologia (GDA) e Tocoginecologia (TMOM), Universidade Federal do Rio Grande do Norte, Natal, RN.

Recebido em 04/09/06 Revisado em 19/01/07 Aceito em 03/04/07 
os parâmetros avaliados, a alteração mais freqüentemente encontrada foi diminuição do HDL-colesterol $(69,6 \%)$, seguida por circunferência da cintura maior que $88 \mathrm{~cm} \quad(57,8 \%)$, hipertrigliceridemia $(31,4 \%)$ e hipertensão arterial $(18,6 \%)$. Tendo em vista o fato de que todos esses marcadores representam fatores de risco independentes para ocorrência de doenças cardiovasculares, confirma-se a hipótese de que as mulheres brasileiras com SOP, a despeito da menor prevalência de SM em relação às americanas, constituem um grupo particular na abordagem em saúde, para quem as estratégias de intervenção devem contemplar não somente o tratamento das repercussões negativas da SOP para a esfera reprodutiva, mas também medidas visando à modificação do perfil de risco cardiovascular.

Acreditamos que os dados apresentados têm importantes aplicações clínicas, podendo ser considerados representativos da morbidade cardiovascular a que essas mulheres estão submetidas. Considerando-se que a SOP pode afetar até $10 \%$ da população feminina em idade reprodutiva e que, com base em nossos resultados, pouco mais de um quarto delas poderá ter associação com SM, denotam-se as sérias implicações para saúde pública e a conseqüente necessidade de rastreamento dos fatores de risco cardiovascular em todas as mulheres com quadro clínico sugestivo da síndrome dos ovários policísticos.

\section{REFERÊNCIAS}

1. Silva RC, Pardini DP, Kater CE. Polycystic ovary syndrome, metabolic syndrome, cardiovascular risk and the role of insulin sensitizing agents. Arq Bras Endocrinol Metab 2006;50:281-90.

2. Vrbikova J, Vondra K, Cibula D, Dvorakova K, Stanicka S, Sramkova D, et al. Metabolic syndrome in young Czech women with polycystic ovary syndrome. Hum Reprod 2005;20:3328-32

3. Carmina E, Napoli N, Longo RA, Rini GB, Lobo RA. Metabolic syndrome in polycystic ovary syndrome (PCOS): lower prevalence in southern Italy than in the USA and the influence of criteria for the diagnosis of PCOS. Eur $J$ Endocrinol 2006;154:141-5.

4. Rotterdam ESHRE/ASRM-Sponsored PCOS Consensus Workshop Group. Revised 2003 consensus on diagnostic criteria and long-term health risks related to polycystic ovary syndrome. Fertil Steril 2004;81:19-25.

5. Executive Summary of the Third Report of the National Cholesterol Education Program (NCEP) Expert Panel on Detection, Evaluation, and Treatment of High Blood Cholesterol in Adults (Adult Treatment Panel III). JAMA 2001;285:2486-97.

6. Oliveira EP, Souza ML, Lima MD. Prevalence of metabolic syndrome in a semi-arid rural area in Bahia. Arq Bras Endocrinol Metab 2006;50:456-65.

7. Hayashida SAY, Halbe HW, Marcondes JAM, Lopes CMC, Barcellos CRG, Rocha MP, et al. Prevalence and predictors of metabolic syndrome in polycystic ovary syndrome. Gynecol Endocrinol 2006;22(supl. 1):235.

Endereço para correspondência:

Tecia Maria Oliveira Maranhão

Programa de Pós-graduação em Ciências da Saúde, UFRN

Rua Gal. Cordeiro de Farias s/n

59012-300 Natal, RN

Fax: (84) 3215-4220

E-mail: tecia@ufrnet.br 